MATHEMATICS OF COMPUTATION

Volume 79, Number 270, April 2010, Pages 983-992

S 0025-5718(09)02314-X

Article electronically published on September 28, 2009

\title{
ON THE EXPONENT OF DISCREPANCIES
}

\author{
GRZEGORZ W. WASILKOWSKI AND HENRYK WOŹNIAKOWSKI
}

\begin{abstract}
We study various discrepancies with arbitrary weights in the $L_{2}$ norm over domains whose dimension is proportional to $d$. We are mostly interested in large $d$. The exponent $p$ of discrepancy is defined as the smallest number for which there exists a positive number $C$ such that for all $d$ and $\varepsilon$ there exist $C \varepsilon^{-p}$ points with discrepancy at most $\varepsilon$. We prove that for the most standard case of discrepancy anchored at zero, the exponent is at most $1.41274 \ldots$, which slightly improves the previously known bound $1.47788 \ldots$. For discrepancy anchored at $\vec{\alpha}$ and for quadrant discrepancy at $\vec{\alpha}$, we prove that the exponent is at most $1.31662 \ldots$ for $\vec{\alpha}=[1 / 2, \ldots, 1 / 2]$. For unanchored discrepancy we prove that the exponent is at most $1.27113 \ldots$. The previous bound was $1.28898 \ldots$. It is known that for all these discrepancies the exponent is at least 1 .
\end{abstract}

\section{INTRODUCTION}

Various discrepancies have been extensively studied in number theory and numerical analysis, see, e.g., 2, 5, 7, 10, 11, 12, 16, 17, 18, 19, 25], and theoretical computer science, see, e.g., [3, 4] and the references given there. In this paper, we study the so-called $B$-discrepancies in the $L_{2}$-norm which measure how well the volumes of certain sets $B(\boldsymbol{t})$ can be approximated knowing only which of the $n$ pre-specified points $\boldsymbol{t}_{j}$ belong to the set $B(\boldsymbol{t})$. The sets $B(\boldsymbol{t}) \subseteq \mathbb{R}^{d}$ are parameterized by vectors $t$ whose number of components depends on $d$; see 14. For specific definitions of $B(\boldsymbol{t})$ we obtain the most standard discrepancy anchored at zero, as well as the discrepancy anchored at $\vec{\alpha}$, the quadrant discrepancy anchored at $\vec{\alpha}$, and the unanchored discrepancy.

The main discrepancy problem is to find $n$ points that minimize discrepancy for the $d$-dimensional case. There are many deep theoretical results pertaining to optimal bounds on how fast discrepancy tends to zero as $n$ tends to infinity. These bounds are usually weak if $n$ is not sufficiently large relative to $d$. For instance, a celebrated result due to Roth [16, 17] and Frolov [6] provides a sharp asymptotic bound of order $n^{-1}[\ln n]^{(d-1) / 2}$ on the discrepancy anchored at zero. However, this bound is an increasing function of $n$ for $n \leq e^{(d-1) / 2}$.

We believe that for large values of $d$, the exponent of discrepancy provides more practical information on the behavior of minimal discrepancies. It is defined as follows. Letting $n^{B}(\varepsilon, d)$ be the smallest number of points for which the corresponding

Received by the editor April 26, 2008 and, in revised form, March 4, 2009.

2000 Mathematics Subject Classification. Primary 41A55; Secondary 11K38.

Key words and phrases. Discrepancy, multivariate integration.

The first author was supported in part by NSF Grant DMS-0609703.

The second author was supported in part by NSF Grant DMS-0608727.

(C)2009 American Mathematical Society Reverts to public domain 28 years from publication 
$B$-discrepancy does not exceed $\varepsilon$, we want to find the smallest number $p^{B}$ such that

$$
n^{B}(\varepsilon, d) \leq C \varepsilon^{-p^{B}} \quad \text { for all } d \geq 1 \text { and } \varepsilon>0 .
$$

Such a number is called the exponent of B-discrepancy.

The main result of this paper is to provide an upper bound on the exponent $p^{B}$. In particular, we show that

- $p^{B} \leq 1.41274 \ldots$ for the $L_{2}$ discrepancy anchored at 0 ,

- $p^{B} \leq 1.31662 \ldots$ for the $L_{2}$ discrepancy anchored at $\vec{\alpha}$ as well as for the quadrant discrepancy anchored at $\vec{\alpha}$ for $\vec{\alpha}=[1 / 2, \ldots, 1 / 2]$,

- $p^{B} \leq 1.27113 \ldots$ for the $L_{2}$ unanchored discrepancy.

It is known that for all the discrepancies mentioned above, the exponent is at least 1. For the discrepancy anchored at zero, the exponent is at least 1.0669; see 9]. The previous upper bounds on the exponent of discrepancy anchored at 0 and unanchored discrepancy were derived in [24] and they were $1.47788 \ldots$ and $1.28898 \ldots$. . respectively. Hence, we provide a small improvement of these bounds here, although their exact values are still unknown.

We briefly comment on the proof technique. As in [24, we use relations between discrepancy and multivariate integration in the worst case and average case settings, and approximation in the average case setting. More precisely, $B$-discrepancy for the $d$-dimensional case is related to multivariate integration in the worst case setting over a reproducing kernel Hilbert space $H\left(K_{d}^{B}\right)$ whose kernel $K_{d}^{B}$ depends on $B$; see [14. Then we use the known fact that multivariate integration over $H\left(K_{d}^{B}\right)$ is equivalent to multivariate integration in the average case setting with the zero-mean Gaussian measure whose covariance kernel is $K_{d}^{B}$. The next step is to use a relation established in 23 . between multivariate integration and approximation both in the average case setting when we are allowed to use only function values. In [24], we use Smolyak's algorithms for estimating the average case errors for the approximation problem. Here, we use the optimal average case bounds for the approximation problem due to a recent result from [8]. The latter result states that the minimal average case errors of algorithms using function values are essentially the same as the minimal average case errors of algorithms using arbitrary linear functionals. It is known that when we can use arbitrary linear functionals, then the minimal average case errors depend on the truncated trace of the eigenvalues of the corresponding integral operators $W_{d}$; see (2.5). For specific $B$-discrepancies mentioned before, it is relatively easy to compute the eigenvalues since the operator $W_{d}$ is given by a tensor product of univariate operators, and therefore the eigenvalues for the $d$-dimensional case are the product of the univariate eigenvalues. Using results from [13], we know necessary and sufficient conditions for the truncated trace of the eigenvalues for the $d$-dimensional case to be independent of $d$. This finally leads to the bounds on the exponent of $B$-discrepancy. We stress that this proof is non-constructive; i.e., we prove the existence of $n$ points with specific bounds on $B$-discrepancy without constructing them.

\section{BASIC DEFINITIONS AND MAIN RESULT}

Following [14, we briefly recall in this section the definition of B-discrepancy. We also state the main result on the exponent of $B$-discrepancies.

For $d \in \mathbb{N}$ and a function $\kappa: \mathbb{N} \rightarrow \mathbb{N}$, let $D \subseteq \mathbb{R}^{\kappa(d)}$ be a Lebesgue measurable set and $\rho: D \rightarrow \mathbb{R}$ be a probability density function. Let $B$ be a mapping from 
$D$ into the set of measurable subsets of $\mathbb{R}^{d}$ such that the measure of $B(\cdot)$, denoted here by $\operatorname{vol}(B(\cdot))$, is a measurable mapping and

$$
\int_{D}[\operatorname{vol}(B(\boldsymbol{x}))]^{2} \rho(\boldsymbol{x}) \mathrm{d} \boldsymbol{x}<\infty .
$$

For given points $\boldsymbol{t}_{1}, \ldots, \boldsymbol{t}_{n} \in D$ and numbers $a_{1}, \ldots, a_{n} \in \mathbb{R}$, let $\operatorname{disc}(\boldsymbol{x})$ be the error of approximating the volume of $B(\boldsymbol{x})$ by a weighted sum $\sum_{j=1}^{n} a_{j} \mathbf{1}_{B(\boldsymbol{x})}\left(\boldsymbol{t}_{j}\right)$,

$$
\operatorname{disc}(\boldsymbol{x}):=\operatorname{vol}(B(\boldsymbol{x}))-\sum_{j=1}^{n} a_{j} \mathbf{1}_{B(\boldsymbol{t})}\left(\boldsymbol{t}_{j}\right),
$$

where $\mathbf{1}_{B(\boldsymbol{x})}$ stands for the indicator function of the set $B(\boldsymbol{x})$. By B-discrepancy of points $\left\{\boldsymbol{t}_{j}\right\}_{j=1}^{n}$ and coefficients $\left\{a_{j}\right\}_{j=1}^{n}$ (or simply $B$-discrepancy for short) we mean the $L_{2}$-norm of $\operatorname{disc}(\cdot)$, i.e.,

$$
\operatorname{disc}^{B}\left(\left\{\boldsymbol{t}_{j}, a_{j}\right\}_{j=1}^{n}\right):=\left[\int_{D}\left[\operatorname{vol}(B(\boldsymbol{x}))-\sum_{j=1}^{n} a_{j} \mathbf{1}_{B(\boldsymbol{x})}\left(\boldsymbol{t}_{j}\right)\right]^{2} \rho(\boldsymbol{x}) \mathrm{d} \boldsymbol{x}\right]^{1 / 2} .
$$

We illustrate this by the following important example.

Example 2.1 $\left(L_{2}\right.$-discrepancy anchored at 0$)$. Consider $D=[0,1]^{d}, \kappa(d)=d$, $\rho \equiv 1$, and $B(\boldsymbol{x})=[0, \boldsymbol{x}):=\left[0, x_{1}\right) \times \cdots \times\left[0, x_{d}\right)$. This corresponds to one of the most classical discrepancies, the $L_{2}$-discrepancy anchored at 0 . It has been considered in a number of books; see, e.g., [2, 5, 10, 12, 18, 19] and the references therein, especially for equal weights $a_{j}=1 / n$ for which

$$
\operatorname{disc}(\boldsymbol{x})=\prod_{j=1}^{n} x_{j}-\frac{1}{n}\left|\left\{\boldsymbol{t}_{j} \in[0, \boldsymbol{x})\right\}\right| .
$$

For arbitrary weights $a_{j}$ we have

$$
\begin{aligned}
{\left[\operatorname{disc}^{B}\left(\left\{\boldsymbol{t}_{j}, a_{j}\right\}_{j=1}^{n}\right)\right]^{2}=} & 3^{-d}-2^{-d+1} \sum_{j=1}^{n} a_{j} \prod_{k=1}^{d}\left(1-t_{j, k}^{2}\right) \\
& +\sum_{i, j=1}^{n} a_{i} a_{j} \prod_{k=1}^{d}\left(1-\max \left(t_{i, k}, t_{j, k}\right)\right) .
\end{aligned}
$$

By the $n$-th minimal $B$-discrepancy we mean the smallest $B$-discrepancy among all $n$-tuples of points and coefficients, i.e.,

$$
\operatorname{disc}^{B}(n, d):=\inf _{\left\{\boldsymbol{t}_{j}, a_{j}\right\}_{j=1}^{n}} \operatorname{disc}^{B}\left(\left\{\boldsymbol{t}_{j}, a_{j}\right\}_{j=1}^{n}\right) .
$$

The initial $B$-discrepancy is the $B$-discrepancy for $n=0$, which reduces to

$$
\operatorname{disc}^{B}(0, d)=\left[\int_{D}[\operatorname{vol}(B(\boldsymbol{t}))]^{2} \rho(\boldsymbol{t}) \mathrm{d} \boldsymbol{t}\right]^{1 / 2} .
$$

For $\varepsilon \in\left(0, \operatorname{disc}^{B}(0, n)\right]$, let

$$
n^{B}(\varepsilon, d):=\inf \left\{n: \operatorname{disc}^{B}(n, d) \leq \varepsilon\right\}
$$


be the smallest number of points $\boldsymbol{t}_{j}$ for which the $B$-discrepancy is at most $\varepsilon$. We say that the $B$-discrepancy is strongly tractable iff there exist numbers $C$ and $p$ such that

$$
n^{B}(\varepsilon, d) \leq C \varepsilon^{-p} \text { for all } d \geq 1 \text { and } \varepsilon \leq \operatorname{disc}^{B}(0, d) .
$$

Then the exponent of the $B$-discrepancy, $p^{B}$, is the smallest $p$ for which (2.2) holds. That is,

$$
p^{B}:=\inf \{p:(2.2) \text { holds with } p\} .
$$

As shown in [14, $B$-discrepancies are related to integration problems over reproducing kernel Hilbert spaces whose kernel $K_{d}^{B}$ depends on the functions $B$ and $\rho$. We will use this relation in the proof of the main result stated at the end of this section. Here we need to recall from [14 the following facts. Define

$$
D^{B}:=\bigcup_{\boldsymbol{x} \in D} B(\boldsymbol{x}) \subseteq \mathbb{R}^{d}
$$

and $K_{d}^{B}: D^{B} \times D^{B} \rightarrow \mathbb{R}$ by

$$
K_{d}^{B}(\boldsymbol{x}, \boldsymbol{y}):=\int_{D} \mathbf{1}_{B(\boldsymbol{t})}(\boldsymbol{x}) \mathbf{1}_{B(\boldsymbol{t})}(\boldsymbol{y}) \rho(\boldsymbol{t}) \mathrm{d} \boldsymbol{t} \text { for all } \boldsymbol{x}, \boldsymbol{y} \in D^{B} .
$$

Clearly, $K_{d}^{B}$ is symmetric and non-negative definite and, hence, can be regarded as a reproducing kernel. Consider therefore the corresponding reproducing kernel Hilbert space $H\left(K_{d}^{B}\right)$. For a more detailed discussion on reproducing kernel Hilbert spaces we refer the reader to [1, 21. Here we only recall the following basic facts. The space $H\left(K_{d}^{B}\right)$ consists of functions $f: D^{B} \rightarrow \mathbb{R}$. For every $\boldsymbol{t} \in D^{B}$ and $f \in H\left(K_{d}^{B}\right)$, we have

$$
K_{d}^{B}(\cdot, \boldsymbol{t}) \in H\left(K_{d}^{B}\right) \quad \text { and } \quad\left\langle f, K_{d}^{B}(\cdot, \boldsymbol{t})\right\rangle_{H\left(K_{d}^{B}\right)}=f(\boldsymbol{t}) .
$$

Actually, $H\left(K_{d}^{B}\right)$ is the completion of the span of functions $K_{d}^{B}(\cdot, \boldsymbol{t})$ for $\boldsymbol{t} \in D^{B}$.

Due to (2.1), the integral of $K_{d}^{B}(\boldsymbol{x}, \boldsymbol{y})$ is finite,

$$
\int_{D^{B}} \int_{D^{B}} K_{d}^{B}(\boldsymbol{x}, \boldsymbol{y}) \mathrm{d} \boldsymbol{x} \mathrm{d} \boldsymbol{y}=\int_{D}[\operatorname{vol}(B(\boldsymbol{x}))]^{2} \rho(\boldsymbol{x}) \mathrm{d} \boldsymbol{x}<\infty .
$$

We need to assume that the function

$$
h_{d}(\boldsymbol{x})=\int_{D^{B}} K_{d}^{B}(\boldsymbol{x}, \boldsymbol{y}) \mathrm{d} \boldsymbol{y} \quad \text { for all } \boldsymbol{x} \in D^{B}
$$

belongs to $H\left(K_{d}^{B}\right)$. This and (2.3) make the integrals $\int_{D^{B}} f(\boldsymbol{x}) \mathrm{d} \boldsymbol{x}$ well defined for $f \in H\left(K_{d}^{B}\right)$. The relation between $B$-discrepancy and integration will be explained in Section 4.

The embedding operator

$$
\text { Id : } H\left(K_{d}^{B}\right) \rightarrow L_{2}\left(D^{B}\right) \quad \text { with } \quad \operatorname{Id}(f)=f
$$

is continuous, and the operator

$$
W_{d}:=\mathrm{Id}^{*} \mathrm{Id}: H\left(K_{d}^{B}\right) \rightarrow H\left(K_{d}^{B}\right)
$$

is selfadjoint, semi-positive definite, and has a finite trace,

$$
\operatorname{trace}\left(W_{d}\right)=\int_{D^{B}} K_{d}^{B}(\boldsymbol{x}, \boldsymbol{x}) \mathrm{d} \boldsymbol{x}=\int_{D^{B}} \operatorname{vol}(B(\boldsymbol{x})) \mathrm{d} \boldsymbol{x}<\infty .
$$


Clearly,

$$
\left(W_{d} f\right)(\boldsymbol{x})=\int_{D^{B}} f(\boldsymbol{y}) K_{d}^{B}(\boldsymbol{x}, \boldsymbol{y}) \mathrm{d} \boldsymbol{y} .
$$

Let $\left\{\left(\lambda_{d, j}, \eta_{d, j}\right)\right\}_{j=1}^{\infty}$ be the eigenpairs of $W_{d}$ with ordered eigenvalues,

$$
W_{d} \eta_{d, j}=\lambda_{d, j} \eta_{d, j} \quad \text { and } \quad \lambda_{d, j} \geq \lambda_{d, j+1} \quad \text { for all } j \geq 1 .
$$

The finite trace of $W_{d}$ means that $\sum_{j=1}^{\infty} \lambda_{d, j}=\int_{D^{B}} K_{d}^{B}(\boldsymbol{x}, \boldsymbol{x}) \mathrm{d} \boldsymbol{x}<\infty$ and, therefore, $\lambda_{d, j}=\mathcal{O}\left(j^{-r}\right)$ for some $r \geq 1$.

We are ready to state the main result of the current paper.

Theorem 2.2. Suppose there exist positive $C_{d, 0}$ and $p$ such that

$$
\left[\sum_{j=n+1}^{\infty} \lambda_{d, j}\right]^{1 / 2} \leq \frac{C_{d, 0}}{(n+1)^{p}} \text { for all } n \geq 0 .
$$

Then there exists a positive number $C_{p}$ dependent only on $p$ such that

$$
\operatorname{disc}^{B}(2 n, d) \leq \frac{C_{d, 0}}{\sqrt{n}} \cdot \min \left(\frac{1}{\sqrt{2}}, \frac{C_{p}}{(n+1)^{p}} \cdot[\ln (\ln (n+2))]^{p+1 / 2}\right)
$$

for all $n \geq 1$. Moreover, if there exists $\tau \in(0,1)$ such that

$$
\sup _{d \geq 1} \sum_{j=1}^{\infty} \lambda_{d, j}^{\tau}<\infty,
$$

then B-discrepancy is strongly tractable with the exponent $p^{B}$ bounded by

$$
p^{B} \leq 2 \tau .
$$

The proof of this theorem is given in Section 4.

\section{TENSOR PRODUCT KERNELS}

We now specialize Theorem 2.2 to the case of tensor product kernels $K_{d}^{B}$. That is, we assume that for every $d \geq 2$ we have $D_{d}^{B}=D_{1}^{B} \times \cdots \times D_{1}^{B}(d$ times $)$ and

$$
K_{d}^{B}(\boldsymbol{x}, \boldsymbol{y})=\prod_{j=1}^{d} K_{1}^{B}\left(x_{j}, y_{j}\right)
$$

for some reproducing kernel $K_{1}^{B}$. As we will illustrate below, (3.1) holds for a number of important $B$-discrepancies. For such kernels, the operators $W_{d}$ also have a tensor product form and, therefore, their eigenvalues $\lambda_{d, j}$ are products of the eigenvalues of the univariate operator $W_{1}$,

$$
\left(W_{1} f\right)(x)=\int_{D_{1}^{B}} f(y) K_{1}^{B}(x, y) \mathrm{d} y .
$$

Such tensor products have been considered in a number of papers. The following result from [13, Thm.6.6] is especially useful here. Suppose that there exists $\tau^{*}$ such that

$$
\tau^{*} \in(0,1] \quad \text { with } \quad \tau^{*}:=\inf \left\{\tau: \sum_{j=1}^{\infty} \lambda_{1, j}^{\tau} \leq 1\right\} .
$$

Then (2.7) holds for any $\tau>\tau^{*}$, which yields

$$
p^{B} \leq 2 \tau^{*} \text {. }
$$


We summarize this in the following proposition.

Proposition 3.1. If $K_{d}^{B}$ is of the tensor product form (3.1) and the eigenvalues of $W_{1}$ satisfy (3.2), then the $B$-discrepancy is strongly tractable with the exponent

$$
p^{B} \leq 2 \tau^{*} .
$$

Using this proposition, we now derive bounds on the tractability exponents for a number of $B$-discrepancies. For these $B$-discrepancies, multivariate integration is well defined and (2.4) holds. We begin with

Example 3.2 (Discrepancy anchored at 0). It is well known that for $d=1$ we have $K_{1}^{B}(x, y)=\min (x, y)$. The space $H\left(K_{1}^{B}\right)$ consists of absolutely continuous functions vanishing at zero whose first derivatives are in $L_{2}([0,1])$. The eigenvalues of the operator

$$
\left(W_{1} f\right)(x)=\int_{0}^{1} f(y) \min (x, y) \mathrm{d} y
$$

are given by

$$
\lambda_{1, j}=\frac{4}{\pi^{2}(2 j-1)^{2}} \quad \text { for } j=1,2, \ldots
$$

Note that $(2 x-1)^{-2 \tau}$ is convex for $x \geq 1$. For $\tau>1 / 2$, we clearly have

$$
f(\tau):=\sum_{j=1}^{\infty}(2 j-1)^{-2 \tau}=\sum_{j=1}^{k}(2 j-1)^{-2 \tau}+E_{k, \tau},
$$

where $E_{k, \tau}$ is between $\int_{k+1}^{\infty}(2 x-1)^{-2 \tau} \mathrm{d} x$ and $\int_{k+1 / 2}^{\infty}(2 x-1)^{-2 \tau} \mathrm{d} x$, i.e.,

$$
\frac{1}{(4 \tau-2)(2 k+1)^{2 \tau-1}}<E_{k, \tau}<\frac{1}{(4 \tau-2)(2 k)^{2 \tau-1}} .
$$

This truncation allows us to compute numerically $f(\tau)$ with arbitrary precision. Using bisection, we checked that (3.2) holds for $2 \tau^{*} \in(1.412742,1.412746)$. That is,

$$
p^{B} \leq 1.41274 \ldots
$$

Note that this gives a small improvement over the bound $1.4778 \ldots$ obtained in [24].

Example 3.3 (Discrepancy anchored at $\vec{\alpha}$ ). As in the previous example, we have $D=[0,1]^{d}, \kappa(d)=d$, and $\rho \equiv 1$. For the anchor $\vec{\alpha}=\left[\alpha_{1}, \ldots, \alpha_{d}\right] \in[0,1]^{d}$, we take

$$
B(\boldsymbol{x})=\left[\min \left(\alpha_{1}, x_{1}\right), \max \left(\alpha_{1}, x_{1}\right)\right) \times \cdots \times\left[\min \left(\alpha_{d}, x_{d}\right), \max \left(\alpha_{d}, x_{d}\right)\right) .
$$

For simplicity, we now assume that $\alpha_{j}=\alpha \in(0,1)$ for all $j$. (Note that $\alpha=0$ corresponds to the previous example, and the case of $\alpha=1$ is equivalent to $\alpha=0$.) Then

$$
K_{1}^{B}(x, y)= \begin{cases}\min (x, y) & \text { if } x, y<\alpha, \\ 1-\max (x, y) & \text { if } x, y \geq \alpha, \\ 0 & \text { otherwise. }\end{cases}
$$

The eigenvalues of $W_{1}$ are obtained in the standard way by double differentiation of the equation

$$
\int_{0}^{1} f(y) K_{1}^{B}(x, y) \mathrm{d} y=\lambda f(x) .
$$


This leads to the equation $-f(x)=\lambda f^{\prime \prime}(x)$ with the boundary conditions $f(\alpha)=$ $f^{\prime}(0)=0$ for $x \in[0, \alpha]$, and $f(\alpha)=f^{\prime}(1)=0$ for $x \in[\alpha, 1]$. In this way we obtain that the eigenvalues of $W_{1}$ are given by

$$
\left\{\lambda_{j}\right\}_{j=1}^{\infty}=\left\{\frac{4 \alpha^{2}}{\pi^{2}(2 k-1)^{2}}\right\}_{k=1}^{\infty} \cup\left\{\frac{4(1-\alpha)^{2}}{\pi^{2}(2 k-1)^{2}}\right\}_{k=1}^{\infty} .
$$

We computed approximations to $2 \tau^{*}$ in a similar way as in the previous example, and we obtained for $\alpha=1 / 2$ that $2 \tau^{*} \in(1.3166258,1.3166259)$. That is

$$
p^{B} \leq 1.31662 \ldots
$$

Example 3.4 (Quadrant discrepancy anchored at $\vec{\alpha}$ ). As before $D=[0,1]^{d}, \kappa(d)=$ $d$, and $\rho \equiv 1$. For $\vec{\alpha}=\left[\alpha_{1}, \ldots, \alpha_{d}\right]$, we take

$$
B(\boldsymbol{x})=\left[w_{1}\left(x_{1}\right), z_{1}\left(x_{1}\right)\right) \times \cdots \times\left[w_{d}\left(x_{d}\right), z_{d}\left(x_{d}\right)\right),
$$

where $\left[w_{j}(x), z_{j}(x)\right)$ equals $[0, x)$ if $x<\alpha_{j}$, and $[x, 1)$ otherwise. For simplicity we take $\alpha_{j}=\alpha$ and then

$$
K_{1}^{B}(x, y)=\frac{1}{2}(|x-\alpha|+|t-\alpha|-|x-t|) .
$$

It can be verified that the operator $W_{1}$ has the same eigenvalues as in the case of discrepancy anchored at $\vec{\alpha}$. Hence, for $\alpha_{j}=1 / 2$ we have

$$
p^{B} \leq 1.31662 \ldots
$$

Example 3.5 (Unanchored discrepancy). We now have $D=\left\{[\boldsymbol{x}, \boldsymbol{y}] \in[0,1]^{2 d}\right.$ : $x_{j} \leq y_{j}$ for $\left.j=1, \ldots, d\right\}, \kappa(d)=2 d, \rho \equiv 1$, and take

$$
B([\boldsymbol{x}, \boldsymbol{y}])=[\boldsymbol{x}, \boldsymbol{y})=\left[x_{1}, y_{1}\right) \times \cdots \times\left[x_{d}, y_{d}\right) .
$$

Then

$$
K_{1}^{B}(x, y)=\min (x, y)-x y
$$

The eigenvalues of $W_{1}$ are obtained as indicated earlier (this time the boundary conditions are $f(0)=f(1)=0$ ) and are given by

$$
\lambda_{1, j}=(\pi j)^{-2} \text { for } j=1,2, \ldots .
$$

As before, we obtained the following estimate: $2 \tau^{*} \in(1.27110,1.27113)$ and hence

$$
p^{B} \leq 1.27113 \ldots
$$

\section{Proof of Theorem 2.2}

As already mentioned, the proof uses, in particular, the relation between $B$ discrepancies and the worst case errors for integration problems defined over the spaces $H\left(K_{d}^{B}\right)$.

More precisely, for given $\left\{\boldsymbol{t}_{j}, a_{j}\right\}_{j=1}^{n}$, consider a linear algorithm

$$
Q_{\left\{\boldsymbol{t}_{j}, a_{j}\right\}_{j=1}^{n}}(f):=\sum_{j=1}^{n} a_{j} f\left(\boldsymbol{t}_{j}\right)
$$

for approximating integrals $\int_{D^{B}} f(\boldsymbol{x}) \mathrm{d} \boldsymbol{x}$. Its worst case error is defined by

$$
e^{\text {wor }}\left(Q_{\left\{\boldsymbol{t}_{j}, a_{j}\right\}_{j=1}^{n}}, \operatorname{INT}_{H\left(K_{d}^{B}\right)}\right):=\sup _{\|f\|_{H\left(K_{d}^{B}\right)} \leq 1}\left|\int_{D^{B}} f(\boldsymbol{x}) \mathrm{d} \boldsymbol{x}-Q_{\left\{\boldsymbol{t}_{j}, a_{j}\right\}_{j=1}^{n}}(f)\right| .
$$


Let $e^{\text {wor }}\left(n, d, \mathrm{INT}_{H\left(K_{d}^{B}\right)}\right)$ denote the $n$-th minimal worst case error, i.e.,

$$
e^{\mathrm{wor}}\left(n, d, \mathrm{INT}_{H\left(K_{d}^{B}\right)}\right):=\inf _{\left\{\boldsymbol{t}_{j}, a_{j}\right\}_{j=1}^{n}} e^{\mathrm{wor}}\left(Q_{\left\{\boldsymbol{t}_{j}, a_{j}\right\}_{j=1}^{n}}, \operatorname{INT}_{H\left(K_{d}^{B}\right)}\right) .
$$

We know from [14 that

$$
\operatorname{disc}^{B}(n, d)=e^{\text {wor }}\left(n, d, \mathrm{INT}_{H\left(K_{d}^{B}\right)}\right) .
$$

Hence, to estimate the $B$-discrepancies, it is enough to estimate the $n$-th minimal worst case errors for the corresponding integration problem.

Due to (2.3), the reproducing kernel $K_{d}^{B}$ has a finite trace and can be viewed as the covariance kernel of a zero-mean Gaussian measure $\mu^{B}$. Consider therefore the integration problem in the average case setting in which the error of $Q_{\left\{\boldsymbol{t}_{j}, a_{j}\right\}_{j=1}^{n}}$ is measured by

$$
e^{\operatorname{avg}}\left(Q_{\left\{\boldsymbol{t}_{j}, a_{j}\right\}_{j=1}^{n}}, \mathrm{INT}_{\mu^{B}}\right)=\left[\mathbb{E}_{\mu^{B}}\left(\int_{D^{B}} f(\boldsymbol{x}) \mathrm{d} \boldsymbol{x}-Q_{\left\{\boldsymbol{t}_{j}, a_{j}\right\}_{j=1}^{n}}(f)\right)^{2}\right]^{1 / 2},
$$

where $\mathbb{E}_{\mu^{B}}$ denotes the expectation with respect to the measure $\mu^{B}$. For more discussions on the average case setting, see, e.g., [15, 20. It is well known that for any algorithm we have

$$
e^{\operatorname{avg}}\left(Q_{\left\{\boldsymbol{t}_{j}, a_{j}\right\}_{j=1}^{n}}, \mathrm{INT}_{\mu^{B}}\right)=e^{\mathrm{wor}}\left(Q_{\left\{\boldsymbol{t}_{j}, a_{j}\right\}_{j=1}^{n}}, \mathrm{INT}_{H\left(K_{d}^{B}\right)}\right),
$$

as well as

$$
e^{\operatorname{avg}}\left(n, d, \operatorname{INT}_{\mu^{B}}\right)=e^{\text {wor }}\left(n, d, \operatorname{INT}_{H\left(K_{d}^{B}\right)}\right),
$$

where $e^{\text {avg }}\left(n, d, \mathrm{INT}_{\mu^{B}}\right)$ denotes the $n$-th minimal error in the average case setting, i.e.,

$$
e^{\operatorname{avg}}\left(n, d, \mathrm{INT}_{\mu^{B}}\right):=\inf _{\left\{\boldsymbol{t}_{j}, a_{j}\right\}_{j=1}^{n}} e^{\operatorname{avg}}\left(Q_{\left\{\boldsymbol{t}_{j}, a_{j}\right\}_{j=1}^{n}}, \mathrm{INT}_{\mu^{B}}\right) .
$$

This is why it is enough to estimate the latter $n$-th minimal error. For that purpose, consider the problem of approximating functions $f$ with the error measured in the $L_{2}$-norm. We will refer to it as the $L_{2}$-approximation problem. It is well known, see [22, that in the average case setting we can consider only linear algorithms of the form

$$
A_{\left\{\boldsymbol{t}_{j}, q_{j}\right\}_{j=1}^{n}}(f)=\sum_{j=1}^{n} f\left(\boldsymbol{t}_{j}\right) q_{j},
$$

where now the $q_{j}$ are functions from the $L_{2}=L_{2}\left(D^{B}\right)$ space. Let $e^{\text {avg }}\left(n, d, \operatorname{APP}_{\mu^{B}}\right)$ be the corresponding $n$-th minimal error,

$$
e^{\operatorname{avg}}\left(n, d, \mathrm{APP}_{\mu^{B}}\right):=\inf _{\left\{\boldsymbol{t}_{j}, q_{j}\right\}_{j=1}^{n}}\left[\mathbb{E}_{\mu^{B}}\left(\left\|f-A_{\left\{\boldsymbol{t}_{j}, a_{j}\right\}_{j=1}^{n}}(f)\right\|_{L_{2}}^{2}\right)\right]^{1 / 2} .
$$

The $n$-th minimal error defined above is important also for the integration problem since, as shown in [23, it yields the following upper bound on the $n$-th minimal error for the integration problem,

$$
e^{\mathrm{avg}}\left(2 n, d, \mathrm{INT}_{\mu^{B}}\right) \leq \frac{1}{\sqrt{n}} e^{\mathrm{avg}}\left(n, d, \mathrm{APP}_{\mu^{B}}\right) .
$$

Hence, to complete the proof we only need to know that the following inequality holds:

$$
e^{\operatorname{avg}}\left(n, d, \mathrm{APP}_{\mu^{B}}\right) \leq \frac{C_{d, 0} C_{p}}{(n+1)^{p}}[\ln (\ln (n+2))]^{p+1 / 2} .
$$


Such an inequality is proved in [8], p. 490, which completes the proof of (2.6).

The second part of the theorem follows from the following argument; see also [13. Thm. 6.1]. Of course,

$$
\lambda_{d, n}^{\tau} \cdot n \leq M_{\tau}:=\sup _{d \geq 1} \sum_{j=1}^{\infty} \lambda_{d, j}^{\tau}
$$

which implies that

$$
\sum_{j=n+1}^{\infty} \lambda_{d, j} \leq M_{\tau}^{1 / \tau} \sum_{j=n+1}^{\infty} j^{-1 / \tau} \leq \frac{M_{\tau}^{1 / \tau}}{1 / \tau-1} n^{1-1 / \tau} .
$$

Since we can take

$$
C_{d, 0}=\left[M_{\tau}^{1 / \tau} /(1 / \tau-1)\right]^{1 / 2} \quad \text { and } \quad p=\frac{1}{2 \tau}-\frac{1}{2}
$$

we get

$$
\operatorname{disc}^{B}(2 n, d)=\mathcal{O}\left(\frac{[\ln \ln (n+1)]^{p+1 / 2}}{n^{1 /(2 \tau)}}\right)
$$

with the factor in the $\operatorname{big} \mathcal{O}$ notation independent of $n$ and $d$. Therefore $p^{B} \leq 2 \tau$, as claimed. This completes the proof of the theorem.

\section{ACKNOWLEDGMENT}

We would like to thank Erich Novak for valuable comments.

\section{REFERENCES}

1. N. Aronszajn, Theory of reproducing kernels, Trans. Amer. Math. Soc. 68 (1950), 337-404. MR 0051437(14:479c)

2. J. Beck and W. W. Chen, "Irregularities of Distributions," Cambridge Tracts in Mathematics 89, Cambridge University Press, 1987. MR.903025 (88m:11061)

3. B. Chazelle, "The Discrepancy Method: Randomness and Complexity." Cambridge Univ. Press, 2002. MR.1779341 (2002c:68002)

4. D. P. Dobkin and D. P. Mitchell, "Random-Edge Discrepancy of Supersampling Patterns," Graphics Interface '93, York, Ontario, 1993.

5. M. Drmota and R. F. Tichy, "Sequences, Discrepancies and Applications," Lecture Notes in Math. 1651, Springer-Verlag, Berlin, 1997. MR1470456 (98j:11057)

6. K. K. Frolov, Upper bounds on discrepancy in $L_{p}, 2 \leq p \leq \infty$, Dokl. Akad. Nauk SSSR 252 (1980), 805-807. MR580823 (81k:10087)

7. F. J. Hickernell, A generalized discrepancy and quadrature error bound, Math. Comp. 67 (1998), 299-322. MR.1433265 (98c:65032)

8. F. J. Hickernell, G. W. Wasilkowski, H. Woźniakowski, Tractability of linear multivariate problems in the average case setting, in "Monte Carlo and Quasi-Monte Carlo Methods 2006," (A. Keller, S. Heinrich, H. Niederreiter, eds.), pp. 359-381, Springer, 2008. MR 2479240

9. J. Matoušek, The exponent of discrepancy is at least 1.0669, J. of Complexity 14 (1998), 448-453. MR1659020 (2000k:65245)

10. J. Matoušek, "Geometric Discrepancy," Algorithms and Combinatorics 18, Springer-Verlag, 1999. MR.1697825 (2001a:11135)

11. H. Niederreiter, Quasi-Monte Carlo methods and pseudo-random numbers, Bull. Amer. Math. Soc. 84 (1978), 957-1041. MR508447 (80d:65016)

12. H. Niederreiter, "Random Number Generation and quasi-Monte Carlo Methods," CBMS-NSF Reg. Conf. Series Appl. Math., Vol. 63, SIAM, Philadelphia, 1992. MR1172997 (93h:65008)

13. E. Novak and H. Woźniakowski, "Tractability of Multivariate Problems," European Mathematical Society, Vol. 6, Zürich, 2008. MR2455266

14. E. Novak and H. Woźniakowski, $L_{2}$ discrepancy and multivariate integration, In "Analytic Number Theory - Essays in Honor of Klaus Roth," Cambridge University Press, to appear. 
15. K. Ritter, "Average-Case Analysis of Numerical Problems," Lecture Notes in Math. 1733, Springer-Verlag, Berlin, 2000. MR1763973 (2001i:65001)

16. K. F. Roth, On irregularities of distribution, Mathematika 1 (1954), 73-79. MR0066435 $(16: 575 \mathrm{c})$

17. K. F. Roth, On irregularities of distribution, IV, Acta Arith. 37 (1980), 67-75. MR598865 (82f:10063)

18. I. H. Sloan and S. Joe, "Lattice Methods for Multiple Integration," Oxford University Press, 1994. MR 1442955 (98a:65026)

19. S. Tezuka, "Uniform Random Numbers: Theory and Practice," Kluwer Academic Publishers, 1995.

20. J. F. Traub, G. W. Wasilkowski, and H. Woźniakowski, "Information-Based Complexity," Academic Press, New York, 1988. MR 958691 (90f:68085)

21. G. Wahba, "Spline Models for Observational Data," SIAM-NSF Regional Conference Series in Appl. Math., Vol. 59, 1990. MR.1045442 (91g:62028)

22. G. W. Wasilkowski, Information of varying cardinality, J. of Complexity 2 (1986), 204-228. MR $922813(88 \mathrm{~m}: 65099)$

23. G. W. Wasilkowski, Integration and approximation of multivariate functions: Average case complexity with isotropic Wiener measure, Bull. Amer. Math. Soc. 28 (1993), 308-314. Full version in: J. of Approx. 77 (1994), 212-227. MR1184000 (93i:65136)

24. G. W. Wasilkowski and H. Woźniakowski, The exponent of discrepancy is at most $1.4778 \ldots$, Math. Comp. 66, (1997), 1125-1132. MR1397448 (97i:11083)

25. H. Woźniakowski, Average case complexity of multivariate integration, Bull. Amer. Math. Soc. 24 (1991), 185-194. MR.1072015 (91i:65224)

Department of Computer Science, University of Kentucky, Lexington, Kentucky 40506

E-mail address: greg@cs.uky.edu

Department of Computer Science, Columbia University, New York, New York 10027 and Institute of Applied Mathematics, University of Warsaw, 02-097 Warsaw, Poland

E-mail address: henryk@cs.columbia.edu 